\title{
Virtual Reality as a Tool for Large Scale Systems
}

\author{
Vladimír Strakoš ${ }^{1}$, Libor Kavka ${ }^{1, *}$, and Filip Beneš ${ }^{2}$ \\ ${ }^{1}$ College of Logistics, Přerov, Palackého 1381/25,75002 Přerov, Czech Republic \\ ${ }^{2} \mathrm{VŠB}$ - Technical University of Ostrava, Institute of Economics and Control Systems, 17. listopadu \\ 15/2172, 70833 Ostrava, Czech Republic
}

\begin{abstract}
Virtual reality (VR) is a wonderful opportunity of information technology which enables the movement of people in the virtual world. The virtual world, into which a person dives, allows them to forget for some time that they are not in the real world, but right now they are living in an entirely different world with unexpected properties. This idea is the grounds for putting forth a proposal to use the virtual world, which is not a copy of the real world, but it is created from purposefully modified 3D images of technical means and properties of these means. Such a world can then be used to control processes, especially when the space of a virtual world is created from quantities obtained in the real world. The huge importance of creating a virtual world from quantities that we have long been storing lies in the fact that one glance, one flight over the surface of such a world is sufficient to get an overview of the behavior of ultra-largescale systems with a number of monitored quantities. It is the only way to assess, evaluate and control systems presented by a large amount of regularly monitored quantities, which are mutually connected with material and information bonds.
\end{abstract}

\section{Introduction}

We perceive the outside world with our senses and behave according to how the world around us affects us. Either we adapt to it or we affect it in such a way as to live our lives well and therefore to be satisfied with ourselves. Now suppose that the world around us is an area we are responsible for, and we are expected to manage it and maintain it in a very good condition. There are a number of effects on our world, against which we have to protect our area. In other words, we must control such a large-scale system. What does it take to control a large-scale system? The key is to know our area, to monitor it closely and regularly and if something is happening, to take measures against undesirable effects.

If we are in charge of a surface area of several $\mathrm{km}^{2}$, a very acceptable way to maintain an overview of such a large area is to have an airplane or, ideally, a helicopter, and to fly over this area from time to time and check if something is happening. How do we know from a helicopter that something is wrong? Only by comparing an image which is stored in our memory and is not real with the image we are seeing. If there is something in a place

*Corresponding author: libor.kavka@,vslg.cz 
which previously was not there, then we land, we find out the details, evaluate the situation and decide what must be done. Then, we look for a person who will do what we want or what we need.

Now, suppose that we have a device to create a virtual, that is non-existent world in reality. The world, that is the surface of the area of interest, will be created from $\mathrm{z}$ coordinates which illustrate for example average salaries of people in a big city. Then, wearing glasses or surrounded by projected images, we find ourselves over the surface of such an area, which does not actually exist but it is created according to salaries of the inhabitants. Without being told, we do not know over what area we are, what surface it is below us. We will see elevations, hills, mountains, and we will start wondering what it is beneath us. If we learn the fact, e.g. average salaries of residents, then from our position we can see immediately where the rich live, where the middle class live and where the poor are concentrated. We can see the differences, big and small, the size of areas with high income and low income, etc. How do we know how many people with high income there are in an area - many or few people? For such information we put colour marking of population density on the surface we created and thus we get a significantly better overview of how many rich people and how many poor people there are. We will fly there to see both the rich and the poor and then, after returning to our office, we can contemplate how to use the information we gathered to improve the situation [1].

It can be reasonably assumed that during our flying over a landscape of lower and higher salaries improved by colour indicating the density of the population, we forget that we are over a landscape which is not real but created as virtual space. Only after landing we will look around in surprise to see where we are and recollect the world in which we lived for some time and in which we dealt with some critical situations. We can invite experts for the next trip so that they can monitor the whole situation with us and together decide what to do and where. It is an incredible opportunity to use virtual reality as a tool for our decisions on utterly real problems.

Consider, as an example, a nice picture of heat consumption in a particular town in Fig.1. The red lines represent the amount of flowing heat. Beneath the surface of these hills there is actually a hidden group of interesting data influencing the consumption of heat in these areas. We can assume, for example, that those tall formations in the back may represent the consumption of factories with energy-intensive production.

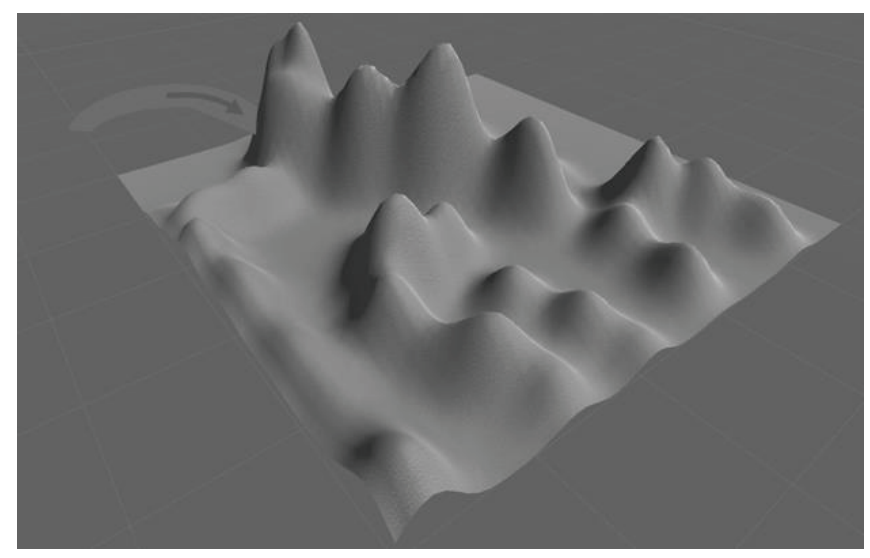

Fig. 1. Example of 3D space representing heat consumption in a mid-size city. Source: authors

On the other hand, in the front on the right there may be a group of houses with a large number of apartments. The valley in the middle may be the city centre surrounded by houses with relatively high consumption. A lot of questions can be derived from such a 
figure and after adding other layers we get very interesting and necessary information for the management of supply and distribution of heat; all this almost "at a glance" in a very short time. This is exactly what present day with a huge amount of information around us requires. Obviously, only an expert can read what is hidden beneath the surface. This is exactly the perfect example of what the authors have promoted all the time and the fact that the model and its properties is important only for experts and not for laymen. A layman can look at a lot of information just as they would look at an image while an expert spends a lot of time studying the model, but they gain a lot of hidden information. Often, it is information they cannot obtain in any other way [1].

From this perspective, our virtual landscape will have the feature of a large-scale model and on this model we can again simulate certain situations over our landscape, we can solve options to find the most favourable measure.

Does it sound complicated? Of course it is very difficult, but when we look, with great admiration, at the ever-expanding possibilities of information technology, we are astonished at the speed with which these options improve. It is very likely that even the creation of an artificial world only from the monitored quantities will be much easier than ever before, and such realization is close ahead. But in order to make use of virtual reality to control, we must perform several difficult follow-up operations.

First, we have to carry out the reduction of our real object onto a system with the individual elements of the system being later displayed as a set of points representing quantities which are available in the various subsystems and throughout the system as well. Then we will pre-think the appearance of the landscape which will be compiled from the selected quantities so that their connections are clear, and we will suggest their location on the surface of the $3 \mathrm{D}$ space that we will be creating. Then all the quantities from the real process will be displayed as $\mathrm{z}$ coordinates of the future $3 \mathrm{D}$ surface. Perhaps the most difficult task will be the fact that the area which we will look at must give us an idea of mutual connections of quantities in the real object. Because we can use colour coding of individual parts, as well as different textures, different characters and spatial objects that complete the displayed area, this is a very difficult task indeed. However, it can be also completed later. Perhaps the last thing to consider is the possibility of placing the elements on the surface, which will allow the control of some quantities through actuators and thus interfere in the real process.

At the end of this introduction, it must be pointed out that we are talking about an artificially created virtual world that is not a copy of the real world. This is an area of constantly improving and well-selling games and that is why we must think about how these great opportunities of information technology can help us in the control of large scale systems.

\section{Real process and control}

We virtually never see real large scale process, which we control, as a whole. Even if we are close to the process, we see only a part and even the view of that part gives us little information. Fig. 2 shows as an example data on the activity of the production process a real company.

Even if we had knowledge of what the individual numbers and characters represent in the real world of a production plant, an overview of the activities of the production process would be very hard to gain and it would be very difficult, if not impossible, to decide about control interventions in this process. 


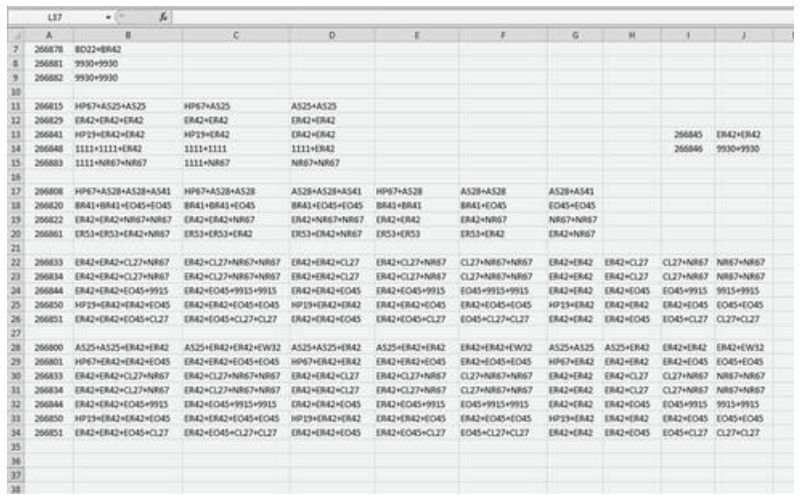

Fig. 2. Example of a screen containing a number of practical operational data, but it is almost impossible to understand this data in order to be able to make decisions. Source: authors

This is one of views which leads the authors to believe that it is much more advantageous to reduce the data into an image or preferably into 3D space, or the space of virtual reality. Consider now as an example the power plant boiler. It is a large object which takes up a lot of space and thus we can always see only a part of it, and even so, we can see its surface and what is essential for us is inside, and this is what we cannot see. Therefore we always reduce the controlled object to a system, we measure important quantities, and these we display on the screen area, for example using SCADA systems, and this is the principle of our view of the use of VR. For the control of - say the above-mentioned boiler, we do not need to see the boiler, we do not even need to know where the sensors are located and what type of sensors they are, but what we need to see is the mutual connections of individual quantities. We do not even need to see a diagram of the boiler, but we need to see the connection between the individual flows of energy, transfer points, the quantity of transferred energy, loss of energy, the amount of fuel which is being withdrawn, the amount of energy transferred to the turbine and the generator etc. Therefore we can view the quantities needed for the control by so far unused display and it is $3 \mathrm{D}$ space.

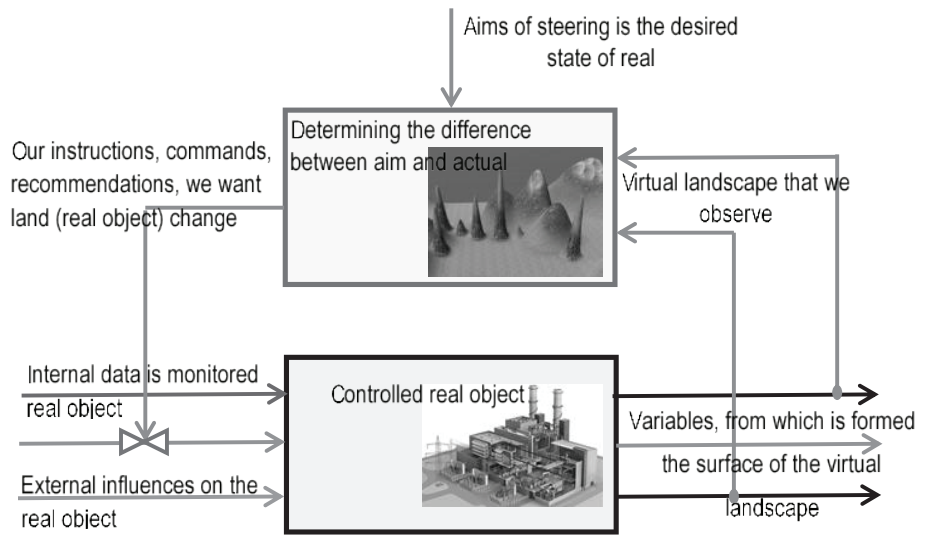

Fig. 3. General control scheme focused on the area of operational and medium-term object control using a $3 \mathrm{D}$ view. Source: authors

Suppose that we control the production process of an object whose information system monitors e.g. 3000 quantities. If we wanted to have an overview of all measured quantities, then we have practically only two ways to display them. One possibility is to create a $50 \times 60$ 
table of measured values. It will not fit in one screen. By grouping several screens we would be able to see the entire table e.g. as in Fig. 2. But then, we have a lot of almost chaotic numbers and it will definitely be hard for us to understand them. What is more, the screen will show us only a situation in one selected point. Computing, however, offers us a number of other ways to study the table, we can create graphs of various structures, we can monitor the development of selected quantities in time, we can use data mining and thus we get information that is hidden in the amount of data and that is not visible without statistics. We have a lot of possibilities, but only a reduction of the data into a properly assembled 3D image will enable us to look at all the data at once, including their mutual connections [2].

The VR environment allows us to display measured quantities in different ways. The easiest possibility, though not very suitable, is to select only one type of quantity that is measured in many places, such as the temperature of the media in some parts of the large scale equipment and to create the surface of the virtual landscape from these values [4].

Thus, we reached a significant fact, namely that we always work with data and information and even though in our case the controlled process is real, we still perceive it with our senses and we put the image of the real world in our brain. If we control such a process, all the decision-making is based on our perceptions and the signals we receive through our senses.

\section{Transforming signals of a real object into the virtual world}

The preparation of the virtual world for control partly follows Fig. 3. The important information for creating a "copy" of the real world is information about the real object. This is important, but for the control of a real object, e.g. a nuclear power plant, the size of cooling towers is not important, but what is important are their properties in relation to the functioning of the entire process. That is, how much heat and from what amount of coolant they are able remove at a certain temperature of the external environment. Their shape, material from which they are built etc. is not so important for the control, but only those values which are needed to fulfil their function, which can be water level, temperature in different locations, functions and state of pumps, resistance of piping systems, etc.

Within the scope of this article we cannot create a detailed analysis of a nuclear power plant in order to create 3D space of virtual reality, but all we want to do is to outline a possible procedure for creating 3D space. Do we really need to create space of virtual reality or is a 3D image sufficient? 3D image is enough, but if this space is put into a virtual reality environment, then we can move freely over this space, we can touch the actuating elements, we can perhaps even watch the space above us, watch the shadows from a certain direction of light, moving objects, etc. [2].

Consider now the quantities essential for creating the 3D space representing the operation of a nuclear power plant. We do not want to create a 3D object of a nuclear power plant because this would be a model of a real object, which would almost useless for control. We are not interested a copy of a real object, but we are interested in the properties and behaviour of a real object, not its shape and size. This is the fundamental difference we are talking about, it is the image suitable for process control what we have in mind.

Fig. 4 shows a beautiful scheme of the primary circuit of a nuclear power plant designed to simulate the plant operation. Such a scheme is very suitable for our thinking. It does not include details which are not significant for the assessment of the operation. But even in this part of the operation many other quantities which are necessary for the control of the primary circuit in real time are measured and checked. The use of virtual reality is not suitable for process control in real time. VR is designed more for the operational and medium-term control and for this the example in fig. 4 is ideal. 


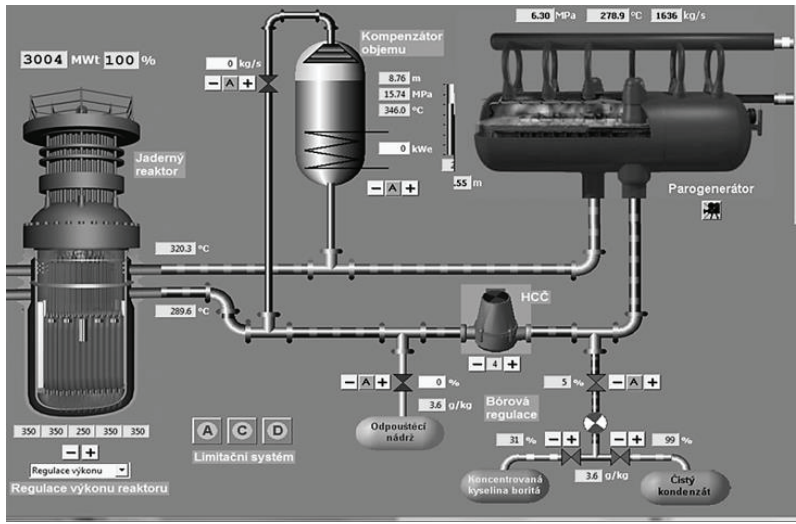

Fig. 4. Scheme of the primary circuit of a nuclear power plant with the most important data. Source: Simulation Program - Information Centre of the Temelín NPP

The reactor is a source of thermal energy, as we can see, a temperature of $320^{\circ} \mathrm{C}$. In this situation the reactor is used for $100 \%$. The thermal energy is transferred through specially treated water in carefully isolated circuit to the steam generator. There the energy is transferred to the water in the secondary circuit, which thus has no direct link with the reactor and the primary circuit and radioactivity does not reach beyond the primary circuit. Volume compensator, which compensates for the change in the volume of water due to the change of heat, is integrated in the circuit. If part of the water in the primary circuit is lost, then condensate and boric acid is added to the primary circuit. For a less informed reader we point out that due to the high pressure in the primary circuit of $15.7 \mathrm{MPa}$, the liquid having a temperature of $350^{\circ} \mathrm{C}$ is still in a liquid state. That is why a liquid flows in the primary circuit while in the secondary circuit, that is behind the steam generator, it is steam. Consider how this scheme will be transformed into 3D space, at the same time making all the important data clearly visible.

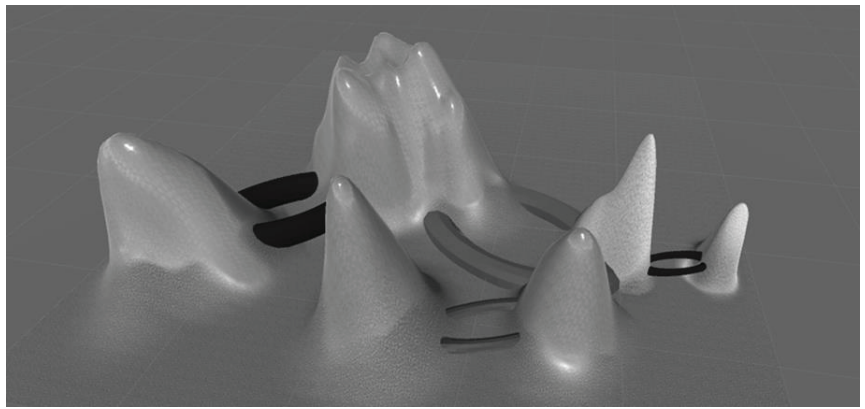

Fig. 5. Scheme of the main energy flows in a nuclear power plant, including several indicators which can be used to check the details in the model. Source: authors

Fig. 5 shows us that it is possible to create a 3D space which would represent, in our case, energy flows in a nuclear power plant, in many variants. It is difficult to say whether this view is the best one. It is certainly not the best, because there are endless possibilities, and therefore the thing is only to create a view that we, senior managers, will like. The thing is not only whether we like it or not, but whether it provides us with enough information that we need to control this process. We would like to point out again that this is not a real-time control, which the control room is in charge of, but it is partly operative control, but mainly it is medium-term control. 
When looking at fig. 5, we will certainly think that it does not make any sense, that we can display this clearly on screen with commonly used tools designed for the construction of schemes of controlled process. After all, the diagram in Fig. 4 proves this, even if it does not show the whole scheme. When we look at both pictures, probably nobody can say which view is clearer.

Thus we come to the essence of the problem and this is namely how large the number of data necessary for the control at a certain control level must be so that it is worth creating a $3 \mathrm{D}$ view. Note the well-arranged space in Fig. 6; this is electric power distribution. This energy system covers a large area of supplied space and apart from that, it indicates a crossborder connection on the right where the energy flow implies significant financial means which "flow" between the connected countries. Additionally, they can sometimes really affect the stability of the whole energy system. The number of end customers, which corresponds to the number of terminal branches, is several million. Such a large number of branches in terms of medium-term control may not even be interesting for us. Consumption of the minor end-user is practically irrelevant in terms of controlling the whole unit. Consumption is, however, significant from a business perspective, because the customer must pay us for the consumed energy. We will focus our control only on larger areas and then display of the distribution of energy consumption in various locations of the monitored area will be very interesting and it will also be necessary for high-quality and economical controlling. 3D space, unlike common ways of display, is irreplaceable for displaying such a move of energy, its sources and places of its consumption and yet we have all the necessary data. Implementation of 3D space compiled from data on the movement of electrical energy can have an almost infinite number of variations [3].

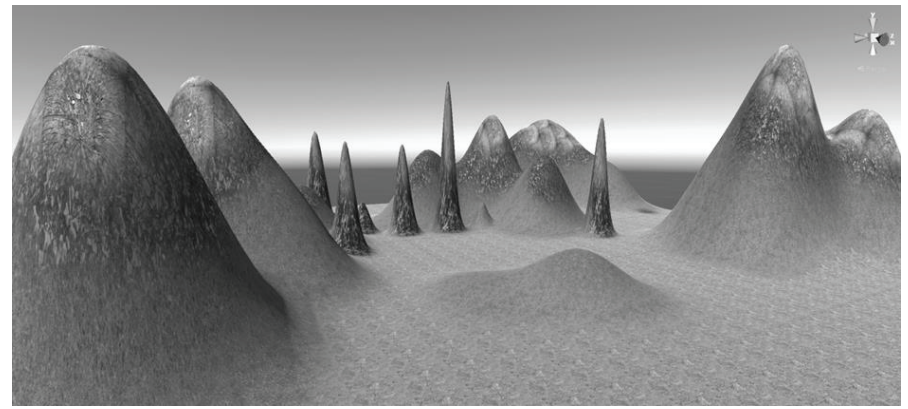

Fig. 6. Part of 3D landscape for displaying the distribution of electrical energy. Source: authors

A simpler variant, which the authors tried to create in Figure 6, will suffice for now. It is loosely based on high voltage distribution systems in the Czech Republic. This means that only significant power plants and some routes of electric line are approximately recorded. This figure, unlike the previous one, is focused on the spatial distribution of the main elements of the entire system for supplying electrical energy to the population. The authors believe that even such a relatively simple representation of the distribution of electrical energy gives a better overview of the whole situation than any other. It will be even better when we make a full use of colour coding of the utilization of electric power distribution, the capacity of power plants, decreases in long distribution lines, cross-border flows, etc. The possibility of using the monitoring panel to "touch" any point in space gives such displays a very interesting overview of the entire system [3].

We can observe what capacity various sources use, what cross-border flows are and the direction of energy flows, how busy the individual line elements are, etc. It would be possible to process more detailed views on the state of switches, transformers, power factor correction etc. in the same way in substations. Of course, one can get all this information in detail on the screen, e.g. in a SCADA system or else, but for a senior manager who takes a 
decision about the situation for a certain period in advance, such a view of the controlled system is certainly more enjoyable and mainly it is free of unnecessary data.

Obviously, the success of such a display depends on the skill and imagination of the creator, and this is indeed one of the major problems with using information 3D space or for other utilization of the possibilities of virtual reality. Some scientific groups dealt with the possibilities of new technologies in control of the technological processes, such as the use of RFID technology, 3D models and use the virtual reality in process control [5].

\section{Conclusions}

The development of information technology brings challenging fast-growing possibilities, which we should take advantage of also for the benefit of the society. Specifically, the issues associated with controlling, as shown in Figure 3, is the part of our activity where we can use technology for the transmission and processing of information. The video game industry, which is constantly developing, is the driving force for the development of the system of representation of the space in which the games take place. These games challenge us to incorporate their possibilities also into the systems control. When considering the ever-increasing transport options for both short and long distances and the increasing speed of transportation, we come to the conclusion that the space for trading and thus also for controlling the movement of goods will continue to expand. It is the development of such technical means which is ideal for the presentation of data and information necessary for the control of movement in a large area. This is the main impulse which led the authors to the fact that it is necessary to prepare possibilities for using the virtual world space for controlling large-scale systems. But in fact, large-scale systems do not have to be large only in terms of space. Consider, for example, the distribution of blood in our body, where there are several billion passages and the failure in any of these, even in the relatively small and insignificant one somewhere in the middle of this network, causes this person major inconvenience, or perhaps even death. This naturally created large-scale supply system is, nevertheless, very well controlled, but it is a question of multi-agent systems and this is a different area of control, emerging again from the development of information technology.

This paper is supported by the research project "From horse-drawn railway to intermodal transport" within Visegrad Fund.

\section{References}

1. S. Aukstakalnis, Old Tappan, Practical augmented reality: a guide to the technologies, applications and human factors for ar and vr, 1st edition, (NJ: Pearson Education, 2016)

2. V. Kebo, O. Kodym, Virtualni realita a rizeni procesu (VSB-TU Ostrava, Czech republic, Montanex, 2011)

3. T. Parisi, Learning virtual reality: developing immersive experiences and applications for desktop, web, and mobile, 1st edition, (Sebastopol, CA: O'Reilly Media, Inc.2011)

4. A. Okita, De Luca, Learning C\# programming with Unity 3D, (Boca Raton: CRC Press, 2015)

5. R. Danel, V. Kohut, O. Kodym, 13th International Multidisciplinary Scientific Geoconference, Information Systems in Coal Preparation Plants and the Use of New Technologies (2013) 